\title{
BMJ Open Effects of the falls and fractures clinic as an integrated multidisciplinary model of care in Australia: a pre-post study
}

\author{
Fernando Gomez, ${ }^{1}$ Carmen Lucia Curcio, ${ }^{1}$ Sharon Lee Brennan-Olsen, ${ }^{\circ, 3}$ \\ Derek Boersma, ${ }^{4}$ Steven Phu, ${ }^{2,3}$ Sara Vogrin, ${ }^{2,3}$ Pushpa Suriyaarachchi, ${ }^{4}$ \\ Gustavo Duque 2,3
}

To cite: Gomez F, Curcio CL, Brennan-Olsen SL, et al. Effects of the falls and fractures clinic as an integrated multidisciplinary model of care in Australia: a pre-post study. BMJ Open 2019;9:e027013. doi:10.1136/ bmjopen-2018-027013

- Prepublication history for this paper is available online. To view these files please visit the journal online (http://dx.doi. org/10.1136/bmjopen-2018027013).

Received 6 0ctober 2018

Revised 7 May 2019

Accepted 1 July 2019

A Check for updates

(c) Author(s) (or their employer(s)) 2019. Re-use permitted under CC BY-NC. No commercial re-use. See rights and permissions. Published by BMJ.

${ }^{1}$ Research Group on Geriatrics and Gerontology, Faculty of Health Sciences, Universidad de Caldas, Manizales, Colombia ${ }^{2}$ Medicine-Western Health, Melbourne Medical School, The University of Melbourne, St Albans, Victoria, Australia ${ }^{3}$ Australian Institute for Musculoskeletal Science (AIMSS), The University of Melbourne and Western Health, St Albans, Victoria, Australia ${ }^{4}$ Sydney Medical School Nepean, University of Sydney, Sydney, New South Wales, Australia

Correspondence to Professor Gustavo Duque; gustavo.duque@unimelb.edu.au

\section{ABSTRACT}

Objectives Traditionally, the approach to fracture prevention has focused on increasing bone mineral density while typically lacking a combined clinical approach to falls prevention and vice versa. To resolve this gap, we implemented and evaluated a novel combined model of care to the assessment and prevention of osteoporosis and falls in the outpatients setting.

Setting Falls and Fractures Clinic (FFC) at Nepean Hospital (Penrith, NSW, Australia).

Participants Pre-effects and posteffects assessment of 106 community-dwelling older patients referred from the community.

Primary and secondary outcome measures Previous falls and fractures were recorded. Clinical, functional and paraclinical evaluations were performed. A comprehensive multidisciplinary care plan was then tailored based on the presence of risk factors. Six-month follow-ups were performed assessing the incidence of falls and fractures, change in risk factors for falls and level of risk, with the recommended plan.

Results We report that $97 \%$ of patients had a fall in the preceding 6 months, $47.6 \%$ of whom experienced a fracture from the fall. Furthermore, $64 \%$ of patients had a marked risk for falling by Physiological Profile Assessment (PPA), 90\% had intermediate-high 10-year probability of fracture according to FRAX and $78 \%$ had sarcopenia. At 6-month follow-up, we observed more than an $80 \%$ reduction in falls and recurrent falls, and $50 \%$ reduction in fractures. In addition, $65 \%$ of patients had reduced PPA and a $57 \%$ reduction in 10 -year fracture probability. Conclusions In conclusion, we suggest that a multidisciplinary FFC can provide substantial reductions in falls and fractures for high-risk older people, even over a relatively short 6 -month time period. The current model of service provision via traditional falls clinics could be significantly improved by encompassing fracture prevention within the multifactorial approach to interventions.

\section{INTRODUCTION}

Multifactorial intervention strategies, based on clinically determined individual needs, can reduce falls and falls risk in older people. ${ }^{1-3}$ The traditional model of geriatrician-run falls clinic for older people is highly

\section{Strengths and limitations of this study}

- We propose a novel operational model of care to specifically include a multifactorial focus on both falls and fracture prevention. We report a significant reduction in incident falls, physiological falls risk, the number of incident fractures and fracture risk.

- Greater patient adherence to recommended interventions, especially a combination of interventions, was more likely associated with reduced falls risk, number of falls and incident fractures.

- Limitations of these study include lack of a control group and the use of self-reported data on falls and adherence.

multifactorial in the provision of (1) assessment and management of patients with falls, mobility and balance problems, (2) time-limited intervention, advice and referral to mainstream services and (3) education and training to caregivers and health professionals. ${ }^{4}$

For older community-dwelling adults, fall prevention strategies may also reduce fall-induced injuries. However, the ability of falls clinics to prevent fractures in high-risk fallers is often contested, ${ }^{5}$ with positive outcomes reported by some, ${ }^{6-8}$ but not all. ${ }^{9} 10$ These discrepancies may be related to the model of care provided by falls clinics, which does usually focus on falls prevention. Likewise, the traditional approach to fracture prevention via osteoporosis/bone metabolic clinics has typically focused on increasing bone mineral density (BMD), while lacking a combined clinical approach to falls prevention. Imperative to the effectiveness of clinics to reduce falls and/or fractures is patient adherence to recommended interventions. ${ }^{211}$ Adherence by older people to a range of falls-prevention interventions is often suboptimal, ranging from $50 \%$ to $70 \% .^{12}$ Similarly, osteoporosis clinics also show significant reductions in 
patient compliance after 6 months of initiating osteoporosis treatment. ${ }^{13}$

In this study, our primary aim was to assess the pre-post effects of a novel combined model of care-the falls and fractures clinic (FFC) - in reducing the incidence of both falls and fractures. Our secondary aims were to evaluate the impact of recommended interventions on reducing falls and fracture risk over 6 months of follow-up.

\section{METHODS}

\section{Setting and participants}

This prospective observational study assessed all community-based patients aged $\geq 65$ years who attended a baseline and 6-month follow-up assessment at our FFC based at Nepean Hospital (Penrith, Australia), from January 2013 to December 2014. Patients had been referred to the FFC by local general practitioners, medical clinics or via the Nepean Hospital inpatient referral processes.

Eligibility criteria to attend the FFC included ability to mobilise with a walker or cane(s), willingness to attend the clinic and at least one of the following: multiple faller $(\geq 2$ in the last year), single faller with established gait and/ or balance problems, unexplained fall/s with apparent complex medical cause(s), a self-reported fragility fracture(s) (last 5 years, and no earlier than 3 months) and a clinically determined increased risk of fractures (for instance, measured by the FRAX or Garvan fracture risk algorithms) and/or a paraclinical measure of femoral neck BMD $\leq 2.5 \mathrm{SD}$ below the young adult mean. Patients with a Mini Mental State Examination (MMSE) less than $18 / 30$ were excluded. Participant consent was waived due to the low nature of this study.

\section{Patient and public involvement}

Patients and public were not involved in the design of this study. Results will not be disseminated to study participants.

\section{Potential confounders}

Age was defined in years. Standing height (centimetres) and weight (kilograms) were used to calculate body mass index (BMI) as $\mathrm{kg} / \mathrm{m}^{2}$. Depression was assessed using the Geriatric Depression Scale, with a cut-off of $<6$ (out of a possible 15 points) considered as normal. ${ }^{14}$ Nutritional intake was assessed using the calculated BMI and the Mini Nutritional Assessment tool, in which a score $<12$ (out of a possible 14) was considered as high risk for malnutrition or undernourishment. ${ }^{15}$

\section{Falls and falls risk}

Patients self-reported the number of falls that were experienced during the 6 months preceding, and following, the first attendance at the FFC, whereby a fall was defined as 'unexpected and involuntary loss of balance, causing the person an undesired contact with the ground'. ${ }^{16}$

\section{Physiological falls risk}

Physiological falls risk was assessed using the Physiological Profile Assessment (PPA) (Prince of Wales Medical
Research Institute, Sydney, Australia), ${ }^{17}$ a valid and reliable tool that can also discriminate between older people who fall once compared with recurrent fallers. Based on the performance in five domains (postural sway, hand reaction time, quadriceps strength, proprioception and edge contrast sensitivity), the PPA provides a standardised fall risk score, with a $75 \%$ predictive accuracy. ${ }^{17}$ PPA z-scores of 0-1 indicate mild risk, 1-2 moderate risk, 2-3 high risk and $\geq 3$ marked risk. ${ }^{18}$ For analyses, and due to the sample size, values of falls risk were categorised as low/mild risk (scores of 0 to $<2$ ), moderate risk ( 2 to $<3$ ) and high risk $(\geq 3)$.

\section{Fear of falling}

The self-reported Survey of Activities and Fear of Falling in the Elderly ${ }^{19}$ was used to assess fear of falling-a major risk factor for falls in ambulatory older persons ${ }^{20}$ - and categorised as no/mild fear (1-2), moderate fear (3) or high fear (4).

\section{Risk factors for falling}

Clinical risk factors for falling ${ }^{4}$ were assessed, including orthostatic hypotension, proprioceptive/vestibular disorder, dizziness, use of medications known to increase falls risk (sedatives, analgesics, psychotropic, antihypertensives, vasodilator or cardiac vasodilator, diuretics, antiparkinsonian, antidepressants, vestibular suppressant and anticonvulsants), vasovagal symptoms or signs, or vision and hearing impairment.

\section{Handgrip strength, gait velocity and sarcopenia}

Grip strength $(\mathrm{kg})$ was recorded as the best of three attempts (Smedley Hand Dynamometer). Using a GAIT Rite (CIR Systems, Havertown, Pennsylvania, USA) instrumented walkway, gait velocity was recorded as the distance (metres) travelled per second. Sarcopenia was determined if at least two of the following criteria were fulfilled: gait velocity $<0.8 \mathrm{~m} / \mathrm{s}$, grip strength $<20 \mathrm{~kg}$ (females) or $<30 \mathrm{~kg}$ (males) and appendicular lean mass (ALM) $/ \mathrm{ht}^{2}<5.5 \mathrm{~kg} /$ $\mathrm{m}^{2}$ (females) or $<7.26 \mathrm{~kg} / \mathrm{m}^{2}$ (males). ${ }^{21}$

\section{Fractures and fracture risk}

Fractures

The number of radiologically confirmed peripheral fracture events, including limbs and limb girdles, was ascertained. ${ }^{16}$ Patients self-reported the number of fractures that were experienced during the 6 months preceding, and following, the first attendance at the FFC.

\section{BMD and body composition by Dual-energy X-ray Absorptiometry}

(DXA)

BMD and body composition (fat and lean mass, and height-adjusted ALM $/ \mathrm{ht}^{2}$ ) were assessed using a Hologic DPX-IQDiscovery DXA machine (GE Healthcare, Pollards Wood, UK). BMD T-scores measured at the femoral neck were classified as: normal (more than -1 SD below the young adult mean), osteopenia (between $-2.4 \mathrm{SD}$ and $-1 \mathrm{SD}$ ) and osteoporosis (at or lower than $-2.5 \mathrm{SD}$ ). 


\section{0-year fracture probability}

The 10-year probability of fracture was assessed using the FRAX fracture risk algorithm (Australian database) ${ }^{22}{ }^{23}$; where available, BMD was included in the calculation of fracture probabilities. Included in the calculation of FRAX scores were patient-specific data regarding secondary osteoporosis, including insulin-dependent diabetes, adult osteogenesis imperfecta, uncontrolled hyperthyroidism, menopause <45years, hypogonadism, chronic malnutrition/malabsorption, chronic liver disease, celiac disease, chronic renal failure, hyperparathyroidism, vitamin D deficiency, hypogonadism, rheumatoid arthritis and glucocorticoid use. Classification of risk, according to FRAX and based on the history of previous osteoporosis treatment, was based on the absolute risk of any major osteoporotic fracture with $20 \%$ considered as high risk, $10 \%-20 \%$ intermediate risk and $<10 \%$ low risk. ${ }^{22}$

\section{Serum measurements}

Blood samples were collected at baseline and 6-month follow-up from patients, while at rest, from which serum concentrations of $25(\mathrm{OH})$ vitamin $\mathrm{D}_{3}$ (vitamin $\mathrm{D}$ ), parathyroid hormone (PTH), calcium, thyroid-stimulating hormone, creatinine, haemoglobin $(\mathrm{Hb})$ and albumin were analysed. Serum vitamin D concentrations were measured by chemiluminescence using the Elecsys $25(\mathrm{OH}) \mathrm{D}_{3}$ assay (Roche): intra-assay and interassay precisions were, respectively, $7.5 \%$ and $10.6 \%$ (normal range $=10-132 \mathrm{nmol} / \mathrm{L})$. Vitamin D deficiency was determined as concentrations $<50 \mathrm{nmol} / \mathrm{L}$. Intact $\mathrm{PTH}$ was measured by immunochemoluminometric assay (Immulite 2000; normal range $=3.9-77.2 \mathrm{pmol} / \mathrm{L}$ : intra-assay and interassay precisions were $7 \%$ and $5 \%$, respectively). Serum calcium and albumin were determined using automated standard laboratory methods and used to correct calcium values (calcium corrected value $=\mathrm{Ca}+0.8$ (40-albumin)) for analyses (normal range $=2.15-$ $2.55 \mathrm{mmol} / \mathrm{L})$. Creatinine clearance was calculated from the Cockcroft formula: (( $(140-$ age in years $) \times$ weight $(\mathrm{kg}) / 72 \times$ creatinine $\mathrm{nmol} / \mathrm{L}))$. Anaemia was categorised as $\mathrm{Hb}<120 \mathrm{~g} / \mathrm{L}$. All measurements were performed at pathology networks affiliated with the Nepean Hospital.

\section{The Nepean FFC}

\section{Baseline assessment}

Following the comprehensive baseline assessment of each patient, the FFC team (composed by a geriatrician, a nurse, a physiotherapist and an occupational therapist) discussed individual falls and fracture risk factors to determine the most appropriate patient-specific multifactorial interventions. This exercise was in line with the Prevention of Falls Network Europe taxonomy whereby subjects receive different combinations of interventions, based on individual risk factors. ${ }^{24}$ The multicomponent interventions, which were tailored but not limited to individually assessed risk factors, ${ }^{25}$ included: vitamin $\mathrm{D} /$ calcium supplementation, osteoporosis medications, adjustment to current medication regimes, supervised group exercise programmes (strength and balance training: two $60 \mathrm{~min}$ sessions per week for 6 weeks), proprioceptive/vestibular retraining exercise, ${ }^{26}$ physical therapy (gait and balance training), ${ }^{25}$ protein supplementation, hip protectors, occupational therapy and referrals to other specialists. These interventions were based on a taxonomy for classifying fall-prevention interventions, as previously recommended. ${ }^{25}$

\section{Six-month follow-up}

At the 6-month follow-up, patients were reassessed for risk factors, incident falls or fractures. Patient data gathered at the 6-month follow-up were ascertained using identical processes and measurement tools as employed for baseline assessments. Patient adherence data during the previous 6 months were also ascertained and defined as completion of the recommended number of home exercise sessions prescribed to the patient at baseline assessment, and/or medication use according to the prescribed timing and dosage. Adherence was defined as follow through with fall-prevention recommendations. ${ }^{27}$ Intervention adherence included personalised education with an emphasis on understanding why adhering to treatment recommendations from the FFC would decrease their risk of falling and/or fracturing. ${ }^{27}$

\section{Statistical analyses}

Differences between baseline assessment and the 6-month follow-up were assessed using Wilcoxon matched-pairs signed rank test. Comparison of the four main outcomes (falls risk, fracture risk, number of falls and fractures) between follow-up and baseline was performed using general estimating equations for ordinal responses (with logit link function) and for counts (with negative binomial link function). Association of other characteristics and interventions on the improvement in the outcome (classified as improved vs not improved) was compared using rank sum test or Fisher's exact test. Change in scores between the adherent versus non-adherent groups were compared using Fisher's exact test. All analyses were performed using Stata V.15.1 (StataCorp. 2017, Stata Statistical Software: Release 15, StataCorp LLC) and $\mathrm{r}$ (R Core Team (2018). R: A language and environment for statistical computing. R Foundation for Statistical Computing, Vienna, Austria. URL https:/ /www.R-project. $\operatorname{org} /$ ).

\section{RESULTS}

Of the 129 patients that attended the baseline FFC assessment, 14 did not undergo a follow-up assessment, while a further 9 were reassessed prior to the 6-month follow-up clinic: this left 106 patients eligible for prospective analyses. No differences were observed for demographics, falls-related or fracture-related outcomes in those that returned for 6-month follow-up compared with those that did not (data not shown). 
Table 1 Participant demographic, clinical and biochemical characteristics at baseline

\begin{tabular}{|c|c|c|c|}
\hline Characteristics & Total $(n=106)$ & Men $(n=34 ; 32 \%)$ & Women $(n=72 ; 68 \%)$ \\
\hline Age, mean (SD) & $78.8(7.0)$ & $77.9(6.6)$ & $79.3(7.2)$ \\
\hline \multicolumn{4}{|l|}{ BMI $\left(\mathrm{kg} / \mathrm{m}^{2}\right), \mathrm{n}=101$} \\
\hline BMI, median (IQR) & $26.0(23.1,30.7)$ & $26.9(24.7,30.1)$ & $25.7(23.0,31.7)$ \\
\hline \multicolumn{4}{|l|}{ BMI categories } \\
\hline Normal (18-24.99) & 40 (39.6) & $8(25.8)$ & $32(45.7)$ \\
\hline Overweight (25-30) & $31(30.7)$ & $15(48.4)$ & $16(22.9)$ \\
\hline Obese $(>30)$ & $30(29.7)$ & $8(25.8)$ & $22(31.4)$ \\
\hline \multicolumn{4}{|l|}{ Falls and falls risk } \\
\hline Number of falls, median (IQR) & $2(1,5)$ & $3(2,6)$ & $2(1,3.5)$ \\
\hline \multicolumn{4}{|l|}{ Physiological falls risk, n (\%) } \\
\hline Mild/moderate risk (1-2) & $2(1.9)$ & $1(2.9)$ & $1(1.4)$ \\
\hline High risk (2-3) & $36(34.0)$ & $12(35.3)$ & 24 (33.3) \\
\hline Marked risk $(\geq 3)$ & $68(64.2)$ & $21(61.8)$ & $47(65.3)$ \\
\hline \multicolumn{4}{|l|}{ Fear of falling, $n(\%)(n=90)$} \\
\hline None/mild (1-2) & $17(18.9)$ & $9(30.0)$ & $8(13.3)$ \\
\hline Moderate (3) & $51(56.7)$ & $14(46.7)$ & $37(61.7)$ \\
\hline High (4) & $22(24.4)$ & $7(23.3)$ & $15(25.0)$ \\
\hline \multicolumn{4}{|l|}{ Sarcopenia, $n=105$} \\
\hline Met criteria for sarcopenia & $83(78.3)$ & $23(69.7)$ & $60(83.3)$ \\
\hline \multicolumn{4}{|l|}{ Fractures and fracture risk } \\
\hline $\mathrm{BMD}^{*}$, median (IQR) $(\mathrm{n}=92)$ & $-2.2(-2.6,-1.2)$ & $-2.15(-2.60,-1.20)$ & $-2.20(-2.70,-1.20)$ \\
\hline \multicolumn{4}{|l|}{ BMD categories, n (\%) } \\
\hline Normal $(>-1)$ & $18(19.6)$ & $5(19.2)$ & $13(19.7)$ \\
\hline Osteopenia (-2.5 to -1$)$ & $41(44.6)$ & $11(42.3)$ & $30(45.5)$ \\
\hline Osteoporosis $(<-2.5)$ & $33(35.9)$ & $10(38.5)$ & $23(34.9)$ \\
\hline \multicolumn{4}{|l|}{ FRAX, n (\%) } \\
\hline Low risk & $12(11.3)$ & $9(26.5)$ & $3(4.2)$ \\
\hline Intermediate risk & $51(48.1)$ & $19(55.9)$ & $32(44.4)$ \\
\hline High risk & $43(40.6)$ & $6(17.6)$ & $37(51.4)$ \\
\hline \multicolumn{4}{|l|}{ Biological measures, median (IQR) } \\
\hline Albumin $(g / L)(n=97)$ & $42(40,44)$ & $41.0(38.0,43.0)$ & $43.0(41.0,45.0)$ \\
\hline Calcium $^{\dagger}(\mathrm{mmol} / \mathrm{L})(\mathrm{n}=92)$ & $2.4(2.3,2.5)$ & $2.3(2.3,2.4)$ & $2.4(2.3,2.5)$ \\
\hline Creatinine $(\mathrm{nmol} / \mathrm{L})(\mathrm{n}=98)$ & $80(65,98)$ & $90.0(75.0,110.0)$ & $76.0(62.5,85.5)$ \\
\hline eGFR (mL/min) (n=96) & $66(53,85)$ & $73.0(55.0,85.0)$ & $65.5(53.0,80.0)$ \\
\hline $\mathrm{Hb}(\mathrm{g} / \mathrm{L})(\mathrm{n}=94)$ & $134(124,141)$ & $134.0(121.0,147.0)$ & $134.0(126.0,140.0)$ \\
\hline Vitamin D (nmol/L) (n=94) & $65(48,84.5)$ & $60.0(48.0,72.0)$ & $67.0(48.0,87.0)$ \\
\hline PTH (pmol/L) $(n=82)$ & $6.2(4.3,8.8)$ & $6.7(4.6,9.0)$ & $5.7(4.2,8.6)$ \\
\hline $\mathrm{TSH}(\mathrm{mlU} / \mathrm{L})(\mathrm{n}=85)$ & $1.4(0.9,2.1)$ & $1.4(1.0,1.9)$ & $1.4(0.8,2.2)$ \\
\hline
\end{tabular}

*Bone mineral density (BMD) T-score is measured at the femoral neck.

† Corrected calcium.

BMI, body mass index; eGFR, estimated glomerular filtration rate; Hb, haemoglobin; PTH, parathyroid hormone; TSH, thyroid-stimulating hormone.

Table 1 presents the overall, and sex-specific, baseline demographic, clinical and biochemical characteristics ( $\mathrm{n}=106 ; 68 \%$ women; mean age $78 \pm 8$ years). A slightly greater proportion of women were obese compared with men (31.4\% vs $25.8 \%$, respectively), while twice as many men were overweight compared with women $(48.4 \%$ and $22.9 \%$, respectively). BMD T-scores at the hip were similar for both sexes, and mainly within the osteopenia range. 
Almost all patients $(97 \%)$ had experienced at least one fall within the 6 months prior to baseline and $47.6 \%$ of patients reported a previous fracture at their first assessment. Approximately $90 \%$ of patients had a FRAX score indicative of intermediate or high risk; more women than men had FRAX scores in the high-risk category $(51 \%$ vs $18 \%)$. Some between-sex differences were observed in albumin and creatinine levels. $\sim 70 \%$ of men and $\sim 85 \%$ of women met the criteria for a diagnosis of sarcopenia.

At the baseline FFC assessment, each patient was recommended at least two interventions; however, the most common number of interventions was four (33\% of the sample), followed by six ( $24 \%$ of patients), and then five interventions $(18 \%)$. The four interventions most often recommended were: supervised group exercise (97.2\%), combined vitamin $\mathrm{D} /$ calcium supplementation (96.2\%), osteoporosis treatment $(67.0 \%)$, and medication adjustment $(66.0 \%)$. At baseline, more than a third of patients were already taking osteoporosis medications (including bisphosphonates, denosumab or teriparatide) and $50 \%$ were already taking vitamin $\mathrm{D} /$ calcium supplementation. Overall, $15 \%$ of patients had adhered to all recommended interventions, $5 \%$ did not adhere to any and $81 \%$ adhered to only some of the recommended interventions. Adherence was greatest for vitamin $\mathrm{D} /$ calcium supplementation $(85 \%)$ and lowest for hip protectors (15.4\%).

Table 2 presents a comparison of falls risk factors from baseline to the 6-month follow-up. Compared with baseline, patients had a reduction in orthostatic hypotension (23.1\% vs $11.5 \%$, respectively) and vasovagal symptoms or signs $(8.0 \%$ vs $1.0 \%$, respectively). Depression and dizziness were marginally reduced $(\mathrm{p}=0.07$, and $\mathrm{p}=0.06$, respectively); however, visual impairment increased from $90.4 \%$ to $96.2 \%$, respectively. Biochemical measures were not available for all patients who attended the follow-up;

\begin{tabular}{|c|c|c|c|c|}
\hline Domain & Data available $n=$ & Baseline & 6-month follow-up & $P$ value \\
\hline \multicolumn{5}{|l|}{ Falls risk } \\
\hline Fear of falling, $n(\%)$ & 40 & & & 0.10 \\
\hline None/mild (1-2) & & $8.0(20.0)$ & $14.0(35.0)$ & \\
\hline Moderate (3) & & $24.0(60.0)$ & $19.0(47.5)$ & \\
\hline High (4) & & $8.0(20.0)$ & $7.0(17.5)$ & \\
\hline \multicolumn{5}{|l|}{ Risk factors for falls } \\
\hline Orthostatic hypotension & 104 & $24(23.1)$ & $12(11.5)$ & 0.002 \\
\hline Depression & 102 & $18(17.7)$ & $12(11.8)$ & 0.07 \\
\hline Proprioceptive ${ }^{*}$ & 103 & $86(83.5)$ & $85(82.5)$ & 1.00 \\
\hline Dizziness & 103 & $26(25.2)$ & $18(17.5)$ & 0.06 \\
\hline Vasovagal symptoms or signs & 100 & $8(8.0)$ & $1(1.0)$ & 0.02 \\
\hline Visual impairment & 104 & $94(90.4)$ & $100(96.2)$ & 0.03 \\
\hline Hearing impairment & 101 & $64(63.4)$ & $63(62.4)$ & 1.00 \\
\hline Malnutrition risk & 101 & $26(25.7)$ & $25(24.8)$ & 1.00 \\
\hline \multicolumn{5}{|l|}{ Sarcopenia } \\
\hline Met criteria ${ }^{\dagger}, \mathrm{n}(\%)$ & 105 & $83.0(78.3)$ & $79.0(74.5)$ & 0.51 \\
\hline \multicolumn{5}{|l|}{ Biochemical measures (IQR) } \\
\hline Albumin (g/L) & 54 & $43(41-45)$ & $42(40-44)$ & 0.046 \\
\hline Corrected calcium (mmol/L) & 41 & $2.4(2.3-2.4)$ & $2.4(2.3-2.4)$ & 0.31 \\
\hline Creatinine (nmol/L) & 54 & $80(70-90)$ & $80(70-95)$ & 0.46 \\
\hline eGFR (mL/min) & 54 & $65.5(56,78)$ & $64(54-82)$ & 0.22 \\
\hline $\mathrm{Hb}(\mathrm{g} / \mathrm{L})$ & 53 & $132(126-142)$ & $132(127-143)$ & 0.30 \\
\hline Vitamin D (nmol/L) & 51 & $63(48-78)$ & $65(54-83)$ & 0.32 \\
\hline PTH (pmol/L) & 43 & $6.9(4.2-9.5)$ & $6.6(4.7-10.8)$ & 0.48 \\
\hline $\mathrm{TSH}(\mathrm{mlU} / \mathrm{L})$ & 44 & $1.5(0.9-2.0)$ & $1.6(0.8-2.1)$ & 0.15 \\
\hline
\end{tabular}

Bold $\mathrm{p}$ values indicate significant difference between baseline and 6-month follow-up measures.

*Proprioceptive and vestibular abnormalities.

†Sarcopenia criterion is fulfilling at least two of the following criteria: gait velocity $<0.8 \mathrm{~m} / \mathrm{s}$, grip strength $<20 \mathrm{~kg}$ (females) and $<30 \mathrm{~kg}$ (males) and appendicular lean mass $/$ height $^{2}<5.5 \mathrm{~kg} / \mathrm{m}^{2}$ (females) or $<7.26 \mathrm{~kg} / \mathrm{m}^{2}$ (males).

$\mathrm{BMD}$, bonemineral density (femoral neck); eGFR, estimated glomerular filtration rate; Hb, haemoglobin; PTH, parathyroid hormone; TSH, thyroid-stimulating hormone. 


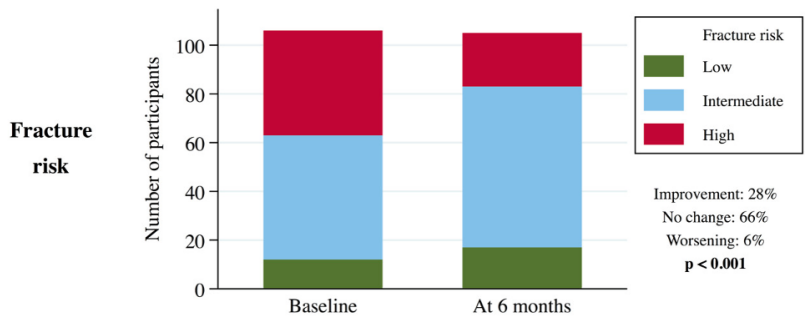

B

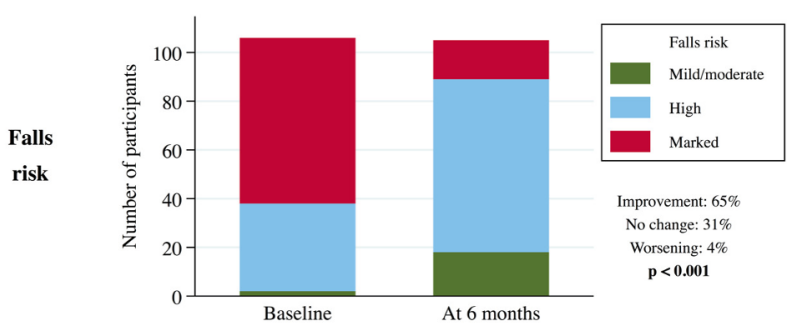

C

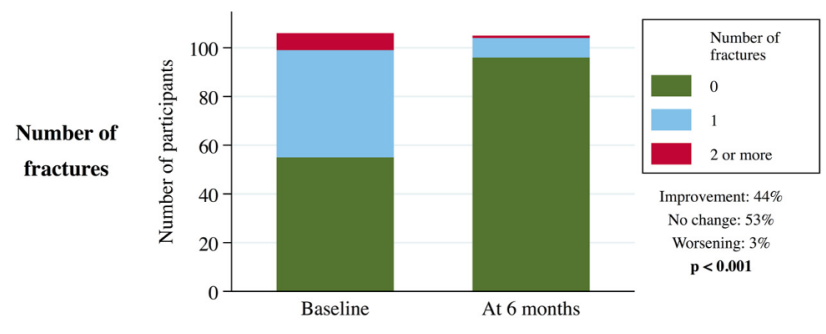

D

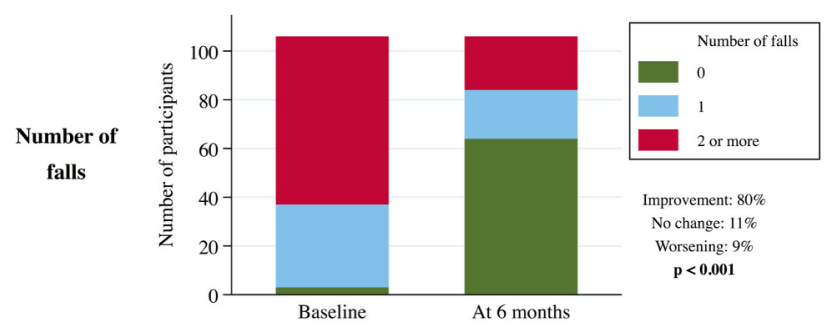

Figure 1 Level of improvement in falls-related and fracturerelated outcomes from baseline to the 6-month follow-up, depending on baseline falls and fracture profiles.

however, a minimal decrease in albumin levels was observed $(\mathrm{p}=0.046)$.

At the 6-month follow-up, $65 \%$ of patients showed reduced physiological falls risk ( $39 \%$ of those initially had moderate risk, and $81 \%$ of those had high risk), and $80 \%$ of patients had a reduced number of falls $(71 \%$ of those with a single fall at baseline had no falls at follow-up, while $55 \%$ of those with $\geq 2$ falls at baseline had no falls at follow-up) (figure 1). The odds of having a lower falls risk at 6 months were 10 times higher compared with baseline (OR 10.0, 95\% CI 5.7 to 17.6, $\mathrm{p}<0.001$ ), while the incidence of falls was lower by $35 \%$ (incident rate ratio (IRR)) $0.65,95 \% \mathrm{CI} 0.58$ to $0.73, \mathrm{p}<0.001$ ).

The incidence of fractures at 6 months was reduced for $76 \%$ (IRR $0.24,95 \%$ CI 0.13 to 0.43 , $\mathrm{p}<0.001$ ) with almost $50 \%$ of patients showing a reduction in the number of fractures. A significant reduction was also observed for the 10-year probability of fracture, calculated by the use of FRAX; $57 \%$ of patients falling into the high-risk group at baseline were classified as either intermediate or low risk at follow-up, and $12 \%$ of those with intermediate FRAX risk at baseline had low risk at follow-up. The odds of having a lower FRAX score at 6 months were two times higher than at baseline (OR 2.23, $95 \%$ CI 1.54 to $3.22, \mathrm{p}<0.001$ ).

Improvement in falls risk and/or number of falls was not associated with any demographic characteristic or intervention received. Higher proportion of those who had an improvement in falls risk showed an improvement in dizziness $(16 \%$ vs $0 \%, \mathrm{p}=0.011)$. Improvement in FRAX was associated with normal BMI (59\% of those with improvement had normal BMI compared with $32 \%$ of those with no improvement, $\mathrm{p}=0.027$ ), prescription of osteoporotic medications $(83 \%$ vs $60 \%, \mathrm{p}=0.024)$ and hip protectors $(60 \%$ vs $27 \%, \mathrm{p}=0.003)$. There were no differences in previous osteoporotic medications $(27 \%$ vs $23 \%, \mathrm{p}=0.80)$. Improvement in number of fractures was associated with female sex $(78 \%$ vs $59 \%, \mathrm{p}=0.058)$, improvement in proprioception $(11 \%$ vs $2 \%, \mathrm{p}=0.039)$ and nutritional risk ( $4 \%$ vs $2 \%, \mathrm{p}=0.004)$. The greatest improvement in patients were observed for those that were prescribed osteoporotic medications (93\% vs $46 \%, \mathrm{p}<0.001)$, protein supplements (33\% vs $12 \%)$ and hip protectors $(59 \%$ vs $19 \%, \mathrm{p}<0.001)$; however, improvements were also seen in patients that were least likely to receive balance exercise (28\% vs 53\%, $\mathrm{p}=0.017$ ). Improvement was not associated with previous osteoporotic medications $(28 \%$ vs $20 \%, \mathrm{p}=0.37$ ).

The comparisons for each of the falls-related and fracture-related outcomes between patients that adhered versus did not adhere for most recommended interventions are presented in table 3. Self-reported adherence to supervised exercise was associated with improvement in at least one category of falls risk $(\mathrm{p}=0.032)$, and a reduced number of falls $(p=0.006)$, while adherence to vitamin $D$ or calcium supplementation and/or osteoporosis medications was associated with a reduction in at least one category of fracture risk ( $\mathrm{p}=0.061$ and $\mathrm{p}=0.018$, respectively). Although proportions were lower, adherence to changes in medications also reduced the number of incident fractures $(\mathrm{p}=0.044)$. No further associations were observed.

\section{DISCUSSION}

In the Nepean FFC, a novel operational model of care to specifically include a multifactorial focus on both falls and fracture prevention, we report a reduction in incident falls, physiological falls risk, the number of incident fractures and in the FRAX risk. Greater patient adherence to recommended interventions, particularly a combination of interventions, was more likely associated with reduced falls risk, number of falls and incident fractures. This observation from the FFC reinforces the positive results from previous pre-post intervention studies that have evaluated outcomes from patients of falls clinics. ${ }^{28}$

The effects of falls clinics to reduce falls have been reported by several studies ${ }^{23829}$; however, the impact on fracture has 
Table 3 Differences between patient adherence versus non-adherence with regards to most recommended interventions, for each of the falls-related and fracture-related outcomes.

\begin{tabular}{|c|c|c|c|c|c|c|c|c|}
\hline \multirow[b]{2}{*}{ Intervention } & \multicolumn{2}{|c|}{ Fractures risk } & \multicolumn{2}{|l|}{ Falls risk } & \multicolumn{2}{|c|}{ Number of fractures } & \multicolumn{2}{|c|}{ Number of falls } \\
\hline & $\begin{array}{l}\text { N (\%) } \\
\text { improved }\end{array}$ & $P$ value & $\begin{array}{l}\text { N (\%) } \\
\text { improved }\end{array}$ & $P$ value & $\begin{array}{l}\text { N (\%) } \\
\text { improved }\end{array}$ & $P$ value & $\begin{array}{l}\text { N (\%) } \\
\text { improved }\end{array}$ & $P$ value \\
\hline Adherent $(n=40)$ & $13(33 \%)$ & 0.65 & $30(77 \%)$ & 0.03 & $18(45 \%)$ & 1.00 & 37 (93\%) & 0.006 \\
\hline Non-adherent $(\mathrm{n}=59)$ & $16(27 \%)$ & & 32 (54\%) & & $25(43 \%)$ & & $41(69 \%)$ & \\
\hline Non-adherent $(n=15)$ & $1(7 \%)$ & & $11(73 \%)$ & & $5(33 \%) 1$ & & $14(93 \%)$ & \\
\hline \multicolumn{9}{|c|}{ Osteoporosis medications $(n=66)$} \\
\hline Adherent $(n=48)$ & $22(46 \%)$ & 0.01 & $34(71 \%)$ & 0.14 & $29(60 \%)$ & 0.56 & 38 (79\%) & 1.00 \\
\hline Non-adherent $(n=18)$ & $2(12 \%)$ & & $8(47 \%)$ & & $12(71 \%)$ & & $15(83 \%)$ & \\
\hline
\end{tabular}

Bold values indicate significant difference between baseline and 6-month follow-up measures.

been little investigated. An evaluation of 13 Australian Falls Clinics showed that the number of fall-induced injuries was reduced by more than $50 \%$ in the 6 months following initial clinical assessment. ${ }^{2}$ Our FFC showed a reduction in self-reported fractures; from $47.6 \%$ of patients who reported a previous fracture at their first assessment, only $8.6 \%$ reported new events at their 6 months of follow-up. Reductions in fractures, although at a much lower level, have been reported by other studies, ${ }^{7}$ while others reported no change in fractures. ${ }^{6830}$ We also observed reductions in fracture probability over the 6 months, as measured by FRAX, despite falls not being included as a major risk factor within the algorithm. While FRAX calculations are recommended as being performed over a 2-year period, ${ }^{22} 31$ we considered that the absolute probability of fracture and the reversible level of risk were important to investigate. We suggest that the observed reduction in FRAX values was most likely related to the effect of our interventions on some of the components of FRAX such as secondary osteoporosis, treatment of rheumatoid arthritis, reduction in alcohol consumption, correction of vitamin D levels, optimisation of diabetes treatment, and so on. The reduction in fractures observed in our model could be explained by a combination of falls-prevention strategies, correction of secondary causes of osteoporosis, and prompt initiation of, and adherence to, osteoporosis treatment. In addition, a continuous reduction in fracture risk would be expected to be potentiated by the antifracture efficacy in those already on osteoporosis medication, which usually starts $6-8$ months after treatment initiation. Notably, the proportion of our patients that were already receiving osteoporosis treatment at baseline $(36 \%)$ was similar to that reported by other studies. ${ }^{30}$ It is probably that many of effects observed 6-month follow-up period were for these patients on treatment at baseline.
Reductions in falls and falls-related injuries are highly associated with clinical interventions, ${ }^{249}$ which could have an ever-stronger beneficial impact would fracture prevention be integrated in the usual model of care. Therefore, it has been previously proposed that falls clinic be operationally linked to osteoporosis services such as FFC, which would facilitate a combined falls and fracture prevention in older persons. $^{32} 33$ Discordant findings from other studies, where no change was observed, may also be related to their patient profile. ${ }^{9}{ }^{10}$ Patients referred to our FFC included those that were overweight, osteosarcopenic, with intermediate/high fracture risk, high/marked risk for falling and moderate/ high fear of falling: a profile similar to that reported for patients attending other falls clinics, ${ }^{2}{ }^{30}$ and the type of patient most likely to benefit from multifactorial interventions. ${ }^{4345}$ Our data reinforce the suggestion of better outcomes for older populations at a higher risk for falls and fractures. Previously, those who had reported exposure to a falls clinic in the past 5 years did report a substantially higher risk of falling in the past 12 months, suggesting it is those at high risk who have undertaken this type of programme. ${ }^{34}$

Sarcopenia is now a recognised risk factor for both falling ${ }^{36}$ and fragility fracture. ${ }^{37}$ In a pilot study, 90 community-dwelling adults aged $\geq 65$ years attending a falls clinic, frailty, sarcopenia and nutritional status were assessed, with a high prevalence of sarcopenia identified. ${ }^{38}$ Patients suffering from both osteoporosis and sarcopenia-defined as osteosarcopenic-are considered at higher risk of poor outcomes such as falls, fractures, frailty and mortality. ${ }^{39}$ Approximately three-quarters of our patients at baseline had sarcopenia (two-thirds of them were osteosarcopenic), and one quarter were at high risk for malnutrition or undernourishment, which is an important risk factor for both osteoporosis and sarcopenia. ${ }^{40}$ Furthermore, the high proportion 
of individuals with obesity and sarcopenia in our study sample support our previous results regarding sarcopenic obese fallers being more likely to have lower BMD, lower grip strength, slower gait velocity and poor balance. ${ }^{41}$ These figures highlight the imperative to diagnose and identify risk factors for sarcopenia in this setting. After assessing nutritional risk, and after recommending protein supplements to all patients at high nutritional risk, we also observed that low adherence to nutritional interventions was associated with reduced albumin at the 6-month follow-up, indicating the importance of reinforcing nutrition as an effective falls and fracture prevention strategy.

The multifactorial recommendations employed by the FFC are comparable to others reported in the literature. ${ }^{26-8} 41$ We report patient adherence of $95 \%$ (full and partial adherence combined), which is mildly superior to other studies, ${ }^{2} 62842$ and, as reported by others, ${ }^{2}$ we also observed patient adherence to vary accordingly across interventions. Strategies aimed at improving patient adherence would further enhance the clinical outcomes of FFCs; these strategies would necessarily involve a patient focus, but should also encompass healthcare providers and healthcare systems. ${ }^{11}$ We speculate there may be several adherence-related reasons for improvements in falls and fracture outcomes in our study. Exercise has been shown to be effective in reducing falls in older adults. ${ }^{1}$ Our results support this evidence and highlight the importance of adherence to exercise programmes. However, data suggest that adherence to home-based falls prevention exercises decline steadily over a 6 -month period. ${ }^{43}$ Given this, healthcare providers have a critical role in improving adherence so as to increase the efficacy of falls prevention programmes. ${ }^{11}$

Data suggest that Fracture Liaison Services, and pharmacist-delivered counselling, may have positive influences on adherence to recommended osteoporosis medications ${ }^{44}$; this is imperative, given that non-adherence to osteoporosis medications has been associated with increased fracture risk over 12 months of follow-up. ${ }^{45}$ However, the reduced 10-year fracture probability that we observed suggests that interventions other than osteoporosis medication may have a positive effect on fracture risk, particularly given the suggestion that only $4 \%-30 \%$ of reduced fracture risk in association with antiresorptive therapy can be attributed to changes in BMD. ${ }^{46}$ Adherence to medications, and changes to medication regimes, appear to positively impact the number of incident fractures: we may speculate reasons for this. First, it may be related to the management of orthostatic hypotension, which is one of the most common interventions prescribed to manage falls risk in clinical practice. ${ }^{11}$ Second, polypharmacy has been shown as increasing the risk of fragility fracture; thus, changes to medication regimes may indirectly result in a decreased risk for falls and thus fractures. ${ }^{47}$

Our study has several strengths. We provide strong evidence that added patient benefit can be gained from traditional geriatrician-run falls clinics, if the model of care is revised to provide a combined focus on falls and fractures. We also provide evidence suggesting the imperative of patient adherence to multifactorial interventions for falls and fracture prevention. Finally, patient adherence at our FFC was higher than previously reported; thus, our FFC model provides novel insights into effective interventions. Our study also has some limitations. Given the absence of a control group and the use of multiple comparisons within the group, we suggest care be taken in interpreting our findings. Future randomised control trials are warranted to support our findings with respect to the observed decrease in falls and fracture risk. Radiographic confirmation of fractures was not undertaken. We acknowledge that self-reported falls, fracture and adherence data, particularly from older persons who are keen to reduce their risk, may introduce social desirability bias. Our study did not employ diaries to record falls and/or fractures. Biochemical data were not available for a large proportion of our FFC patients that attended the 6-month follow-up, thereby limiting our ability to comment on change in biomarkers. Similarly, a longer period of time would be required in order to observe substantial changes in BMD, and indeed to investigate the efficacy of the FFC in populations at high risk for falls and fractures. In addition, a much larger sample is required in order to confirm our observed antifracture reduction, and to enable us to confirm the generalisability of our findings. Finally, investigating the cost effectiveness of our interventions was beyond the scope of this study.

\section{CONCLUSION}

In conclusion, we report that our multidisciplinary FFC substantially reduced falls and fracture risk in older people at high risk of these adverse events, even over a relatively short 6-month time period. The current model of service provision via falls clinics could be significantly improved by encompassing fracture prevention within the multifactorial approach to interventions. This new model of care appears to be the optimal setting to assess and treat older patients with osteosarcopenia at very high risk for falls and fractures. Targeted efforts to improve patient adherence will enhance the benefit gained, especially if adherence to a combination of interventions is achieved.

Acknowledgements The authors would like to thank the Department of Aged Care at Nepean Hospital (Penrith, NSW, Australia) for its support to this project.

Contributors FG, CLC, DB, SP, PS and GD generated, analysed and interpreted the patient data regarding the clinical and pathology results. SV and SLB-0 performed the statistical analysis, and were major contributors in writing the manuscript. All authors read and approved the final manuscript.

Funding This study was funded by a grant from the Australian Institute for Musculoskeletal Science. SLB-0 is supported by a Career Development Fellowship from the National Health and Medical Research Council of Australia (1107510). FG is supported by an Australian Leadership Award Fellowship from Australian Government Overseas Aid Program.

Competing interests None.

Patient consent for publication Not required.

Ethics approval This study was approved by the Nepean Blue Mountains Local Health District Human Ethics Research Committee (Reference number HREC/11/ NEPEAN/94).

Provenance and peer review Not commissioned; externally peer reviewed.

Data sharing statement Data are available upon reasonable request. 
Open access This is an open access article distributed in accordance with the Creative Commons Attribution Non Commercial (CC BY-NC 4.0) license, which permits others to distribute, remix, adapt, build upon this work non-commercially, and license their derivative works on different terms, provided the original work is properly cited, appropriate credit is given, any changes made indicated, and the use is non-commercial. See: http://creativecommons.org/licenses/by-nc/4.0/.

\section{REFERENCES}

1. Gillespie LD, Robertson MC, Gillespie WJ, et al. Interventions for preventing falls in older people living in the community. Cochrane Database Syst Rev 2012;9(9):CD007146.

2. Hill KD, Moore KJ, Dorevitch MI, et al. Effectiveness of falls clinics: an evaluation of outcomes and client adherence to recommended interventions. J Am Geriatr Soc 2008;56:600-8.

3. Tan PJ, Khoo EM, Chinna K, et al. An individually-tailored multifactorial intervention program for older fallers in a middleincome developing country: Malaysian Falls Assessment and Intervention Trial (MyFAIT). BMC Geriatr 2014;14:78.

4. Tinetti ME, Kumar C. The patient who falls: "It's always a trade-off". JAMA 2010;303:258-66.

5. Kannus $P$, Sievänen $H$, Palvanen $M$, et al. Prevention of falls and consequent injuries in elderly people. Lancet 2005;366:1885-93.

6. Perell KL, Manzano ML, Weaver R, et al. Outcomes of a consult fall prevention screening clinic. Am J Phys Med Rehabil 2006;85:882-8.

7. Sze PC, Cheung WH, Lam PS, et al. The efficacy of a multidisciplinary falls prevention clinic with an extended step-down community program. Arch Phys Med Rehabil 2008;89:1329-34.

8. Palvanen M, Kannus P, Piirtola M, et al. Effectiveness of the chaos falls clinic in preventing falls and injuries of home-dwelling older adults: a randomised controlled trial. Injury 2014;45:265-71.

9. Moore M, Williams B, Ragsdale S, et al. Translating a multifactorial fall prevention intervention into practice: a controlled evaluation of a fall prevention clinic. J Am Geriatr Soc 2010;58:357-63.

10. Lord SR, Tiedemann A, Chapman K, et al. The effect of an individualized fall prevention program on fall risk and falls in older people: a randomized, controlled trial. J Am Geriatr Soc 2005;53:1296-304

11. Fortinsky RH, lannuzzi-Sucich M, Baker DI, et al. Fall-risk assessment and management in clinical practice: views from healthcare providers. J Am Geriatr Soc 2004;52:1522-6.

12. Hill KD, Black K, Vrantsidis F, et al. Evaluation of a Falls Clinic and development of a measurement and outcome framework: Review of Movement Disorders Clinic. Report of the Victorian Department of Human Services. Melbourne, Australia: DHS, 2002.

13. Fojas MC, Southerland LT, Phieffer LS, et al. Compliance to The Joint Commission proposed Core Measure set on osteoporosisassociated fracture: review of different secondary fracture prevention programs in an open medical system from 2010 to 2015. Arch Osteoporos 2017;12:16.

14. Wancata J, Alexandrowicz R, Marquart B, et al. The criterion validity of the Geriatric Depression Scale: a systematic review. Acta Psychiatr Scand 2006;114:398-410.

15. Guigoz Y. The Mini Nutritional Assessment (MNA) review of the literature--What does it tell us? J Nutr Health Aging 2006;10:466-85.

16. Lamb SE, Jørstad-Stein EC, Hauer K, et al. Development of a common outcome data set for fall injury prevention trials: the Prevention of Falls Network Europe consensus. J Am Geriatr Soc 2005;53:1618-22.

17. Lord SR, Sherrington C, Menz H. A physiological profile approach for falls prevention. Falls in Older People. Risk factors and strategies for prevention. Cambridge: Cambridge University Press, 2001.

18. Lord SR, Menz HB, Tiedemann A. A physiological profile approach to falls risk assessment and prevention. Phys Ther 2003;83:237-52.

19. Lachman ME, Howland J, Tennstedt S, et al. Fear of falling and activity restriction: the survey of activities and fear of falling in the elderly (SAFE). J Gerontol B Psychol Sci Soc Sci 1998;53:P43-P50.

20. Auais M, Alvarado B, Guerra R, et al. Fear of falling and its association with life-space mobility of older adults: a cross-sectional analysis using data from five international sites. Age Ageing 2017;46:459-65.

21. Cruz-Jentoft AJ, Baeyens JP, Bauer JM, et al. Sarcopenia: European consensus on definition and diagnosis: Report of the European Working Group on Sarcopenia in Older People. Age Ageing 2010;39:412-23.

22. Curtis EM, Moon RJ, Harvey NC, et al. The impact of fragility fracture and approaches to osteoporosis risk assessment worldwide. Bone 2017;104:29-38.
23. Kanis JA, Oden A, Johansson $\mathrm{H}$, et al. FRAX and its applications to clinical practice. Bone 2009;44:734-43.

24. Rimland JM, Abraha I, Dell'Aquila G, et al. Effectiveness of NonPharmacological Interventions to Prevent Falls in Older People: A Systematic Overview. The SENATOR Project ONTOP Series. PLOS One 2016;11:e0161579.

25. Lamb SE, Becker C, Gillespie LD, et al. Reporting of complex interventions in clinical trials: development of a taxonomy to classify and describe fall-prevention interventions. Trials 2011;12:125.

26. Duque G, Boersma D, Loza-Diaz G, et al. Effects of balance training using a virtual-reality system in older fallers. Clin Interv Aging 2013;8:257-63.

27. Gibson K, Greene DP, Sample PL, et al. Fall Prevention: Relatedness of Adherence to Recommendations and Self-Rated Knowledge. Phys Occup Ther Geriatr 2010;28:215-24.

28. Puisieux F, Pollez B, Deplanque D, et al. Successes and setbacks of the falls consultation: report on the first 150 patients. Am J Phys Med Rehabil 2001;80:909-15.

29. de Negreiros Cabral K, Perracini MR, Soares AT, et al. Effectiveness of a multifactorial falls prevention program in community-dwelling older people when compared to usual care: study protocol for a randomised controlled trial (Prevquedas Brazil). BMC Geriatr 2013;13:27

30. Houghton S, Birks V, Whitehead $\mathrm{CH}$, et al. Experience of a falls and injuries risk assessment clinic. Aust Health Rev 2004;28:374-81.

31. Aspray TJ. Fragility fracture: recent developments in risk assessment. Ther Adv Musculoskelet Dis 2015;7:17-25.

32. Gomez F, Curcio CL, Suriyaarachchi P, et al. Differing approaches to falls and fracture prevention between Australia and Colombia. Clin Interv Aging 2013;8:61-7.

33. Demontiero O, Gunawardene P, Duque G. Postoperative prevention of falls in older adults with fragility fractures. Clin Geriatr Med 2014;30:333-47.

34. Hill KD, Day L, Haines TP. What factors influence communitydwelling older people's intent to undertake multifactorial fall prevention programs? Clin Interv Aging 2014;9:2045-53.

35. Bleijlevens $\mathrm{MH}$, Hendriks MR, van Haastregt JC, et al. Process factors explaining the ineffectiveness of a multidisciplinary fall prevention programme: a process evaluation. BMC Public Health 2008;8:332.

36. Landi F, Liperoti R, Russo A, et al. Sarcopenia as a risk factor for falls in elderly individuals: results from the ilSIRENTE study. Clin Nutr 2012;31:652-8.

37. Chalhoub D, Cawthon PM, Ensrud KE, et al. Osteoporotic fractures in men study research group. Risk of nonspine fractures in older adults with sarcopenia, low bone mass, or both. J Am Geriatr Soc 2015;63:1733-40.

38. Thomas S, Miller M, Whitehead C, et al. Falls Clinics: an opportunity to address frailty and improve health outcomes (preliminary evidence). Aging Clin Exp Res 2010;22:170-4.

39. Hirschfeld HP, Kinsella R, Duque G. Osteosarcopenia: where bone, muscle, and fat collide. Osteoporos Int 2017;28:2781-90.

40. Huo YR, Suriyaarachchi P, Gomez F, et al. Comprehensive nutritional status in sarco-osteoporotic older fallers. J Nutr Health Aging 2015;19:474-80.

41. Huo YR, Suriyaarachchi P, Gomez F, et al. Phenotype of sarcopenic obesity in older individuals with a history of falling. Arch Gerontol Geriatr 2016;65:255-9.

42. Hogan DB, MacDonald FA, Betts J, et al. A randomized controlled trial of a community-based consultation service to prevent falls. CMAJ 2001;165:537-44.

43. Morey MC, Pieper CF, Crowley GM, et al. Exercise adherence and 10 -year mortality in chronically ill older adults. J Am Geriatr Soc 2002;50:1929-33.

44. Jaleel A, Saag KG, Danila MI. Improving drug adherence in osteoporosis: an update on more recent studies. Ther Adv Musculoskelet Dis 2018;10:141-9.

45. Mikyas Y, Agodoa I, Yurgin N. A systematic review of osteoporosis medication adherence and osteoporosis-related fracture costs in men. Appl Health Econ Health Policy 2014;12:267-77.

46. Järvinen TL, Sievänen $\mathrm{H}$, Khan KM, et al. Shifting the focus in fracture prevention from osteoporosis to falls. BMJ 2008;336:124-6.

47. Pan HH, Li CY, Chen TJ, et al. Association of polypharmacy with fallrelated fractures in older Taiwanese people: age- and gender-specific analyses. BMJ Open 2014;4:e004428. 